\title{
Phase contrast imaging (PCI) investigation for mouse kidney
}

\author{
Peng Yifeng $^{1}$ (Corresponding author), Zhao Ze hua ${ }^{1}$, Xiao tiqiao ${ }^{2}$, Zhu Peiping ${ }^{3}$, He $\mathrm{Wei}^{3}$ \\ 1) Radiology Department, Putuo Hospital, Shanghai University of traditional chinese medicine, Shanghai 200062, China \\ 2) Shanghai Institute of Applied Physics, Chinese Academy of Sciences, Shanghai 201204, China \\ 3) Institute of High Energy Physics, Chinese Acade of Science, Beijing 100049, China
}

[Abstract] Objective To evaluate the privilege of X-ray phase contrast imaging on the microstructure of mouse kidneys, and explore the imaging differences on kidney images obtained by DEI and in-line outline X-ray imaging (IOXI) with synchrotron radiation (SR) facility. Materials and Methods The SD mouse kidneys are taken as samples. DEI images were brought out at 4W1A beam line of BSRF and the in-line outline images were recorded at BL13W beam line of SSRF. Contrast of soft tissue and spatial resolution were selected mainly to evaluate the image quality. Results The kidney microstructures, which are usually hard to be visualized by conventional radiography, were recorded clearly with both of the imaging modalities. The kidney vessels of about $10 \mu \mathrm{m}$ in diameter were revealed, however more minute structures such as the renal glomeruli, were resolvable only in images taken with IOXI. Conclusion The phase contrast imaging has great potential to reveal the structures inside soft tissues which could achieve images of high quality for the tiny vessels without any administration of contrast media, and more microstructures of the mouse kidneys were demonstrated on IOXI. Due to the sample setup and fine images, the IOXI maybe well better manner in biological tissue imaging study.

Keywords: phase contrast imaging(PCI); in-line outline X-ray imaging(IOXI); diffraction enhanced imaging(DEI); Synchrotron radiation; mouse kidney

Conventional medical X-ray imaging is based on absorption contrast, which relies on the differences in linear attenuation coefficients between the different tissues, producing differences in photon fluxes incident upon the detector. When X-ray penetrates the body, these differences in linear attenuation coefficients are substantial between bone and soft tissue, so the difference between these tissues is obvious and clear. However, if the density variations of these tissues is very small, the difference between these tissues will be too small to be visualized. for instance, it is well known that the kidney is very important organ in biological bodys, however being almost composed of light elements cannot be well visualized. and being denser than the surrounding tissue, they appear as lucencies or blurred structure in conventional technique. The difference between normal and disease are subtle, so that in many cases microscopic examinations by needle core biopsies are needed. due to low resoultion or weak contrast, presently in practice there is no perfect technique to make it well visible. How can we see the difference?

A new imaging mode is to make soft tissue sample visible through the phase information after penetrating samples[1].The complex refractive index, $\mathrm{n}=1-\delta$ - $\mathrm{i} \beta^{[2]}$, can be used to describe the behavior of X-rays traveling through subjects. At typical X-ray energies used in medical radiography, the reflection contrast, arising from changes in the phase-shift term $\delta$, may be one or two orders of magnitude greater than the absorption contrast, which is due to variations of the absorption term $\beta^{[3]}$. This is particularly the situation in light-element samples such as biological tissue sample. We call such method as phase contrast imaging which relies on the phase of the traversing X-rays.

The X-rays phase-contrast imaging technique, which has about a 1000-fold higher sensitivity for detecting the light element, such as hydrogen, carbon, nitrogen, and oxygen than the conventional X-rays absorption -contrast methods, even the biological soft tissues can be visualizated without any contrast agent ${ }^{[4][5][6]}$.

There are usually three PCI manners basing on different imaging mechnisms: diffraction enhanced imaging (DEI) or refraction contrast imaging DEI ${ }^{[7]}$, $\mathrm{IOXI}^{[8]}$ and Interferometric imaging ${ }^{[9]}$.

It is necessary that the imaging light source is of coherent, just like the synchrotron radiation (SR), which uses monochromatic SR as the $\mathrm{X}$-ray source and a high-definition camera or video system as a detector.

Recently some works have be done in SR facility indicated that phase-contrast imaging technique is a promising technique for kidney imaging ${ }^{[10][11][12]}$.

Due to different mechniam, some difference should be exist, how much different imaging results among the manners? we aim to compare and analyze mouse kidney images taken by IOXI and DEI.

\section{Materials and Methods}

The experiments were performed at the 4W1A beam line in the Beijing Synchrotron Radiation Facility (BSRF) and at the BL13W1 line in the Shanghai Radiation Facility(SSRF). The experimental setup are sketched in Fig. 1,2. the experiment parameters are different at two facilitys lines. The wiggler source of the beam line $4 \mathrm{~W} 1 \mathrm{~A}$ in BSRF was positioned $43 \mathrm{~m}$ from the experiment hutch. The electron beam energy in the storage ring was $2.5 \mathrm{GeV}$ with a current of $150 \mathrm{~mA}$. However in SSRF the wiggler source of the beam line BL13W1 was positioned $32 \mathrm{~m}$ from the experiment hutch. The electron beam energy in the storage ring was $3.5 \mathrm{GeV}$ with a current of $200 \mathrm{~mA}$. 
In the SSRF IOXI experiment, only one crystal monochromator was used. The distance between the sample and the detector was tunable, and $105 \mathrm{~cm}$ was selected in this study, the exposure time for each image was $10 \mathrm{~ms}$. while in the DEI experiment in BSRF, two crystals were used, one crystal called monochromator was used to select a small energy band from the incident synchrotron radiation forms the imaging beam which strikes the object; the other crystal called analyser similar to the type used in the monchromator as a scatter rejection optic that diffracted the beam which is transmitted through the object being imaged. a sample investigated was placed between the two crystals. The distance between the sample and the detector was $25 \mathrm{~cm}$ that has been identified as the best imaging distance in many experiments.

The kidney was from SD mouse, the kidneys were extracted quickly and fixed using $10 \%$ formalin. In order to be well compared, the same mouse kidney was used in two manner experiment. The light source is about size of $10 \mathrm{~mm}(\mathrm{H}) \times 3 \mathrm{~mm}(\mathrm{~V})$ in SSRF. in the BSRF DEI imaging, experiment was performed at the X-ray energy of $8 \mathrm{keV}$ with a beam size of $20 \mathrm{~mm}(\mathrm{H}) \times 8 \mathrm{~mm}(\mathrm{~V})$, where $\mathrm{H}$ and $\mathrm{V}$ means horizontal and vertical size. the samples were and fixed on the sample holder.

The X-ray beam, which was $13.3 \mathrm{KeV}$ in the $\mathrm{SSRF}$ and $8 \mathrm{KeV}$ in BSRF, after going through the sample, was detected by an $\mathrm{x}$-ray CCD camera. The images can be recorded using X-ray CCD with the pixel size $10.8 \mu \mathrm{m}$ (Photonic Science Limited, X-ray FDI) in BSRF and with the pixel size $9 \mu \mathrm{m}$ (Photonic-Science VHR) in SSRF respectivily.

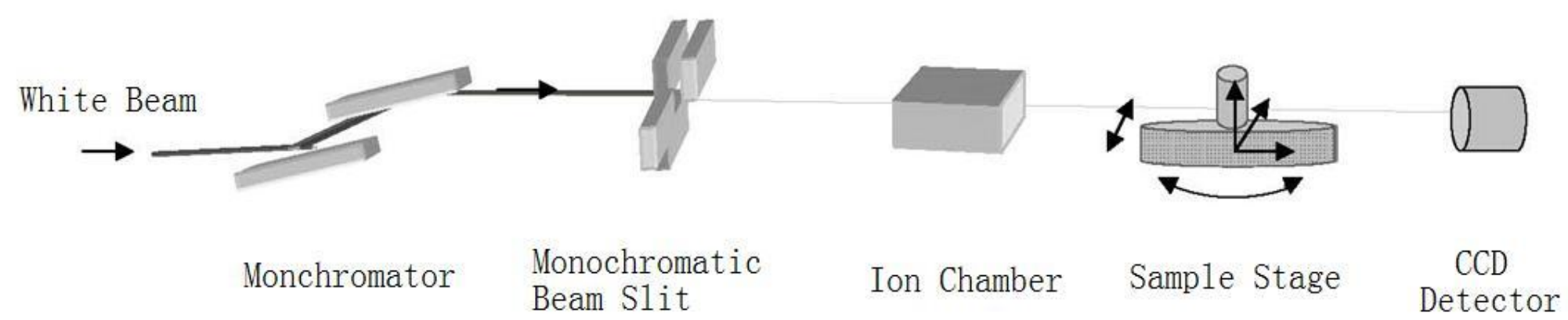

Fig1: SSRF IOXI experimental set-up

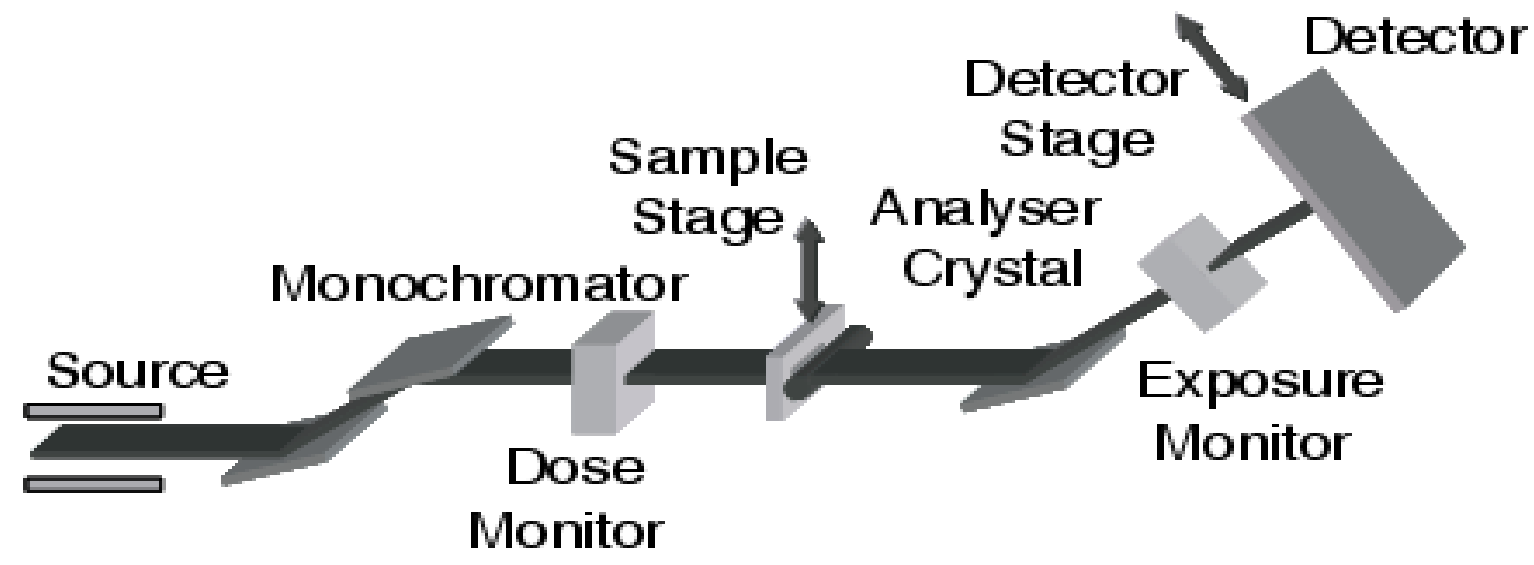

Fig2: BSRF DEI experimental set-up

\section{Results}

1)IOXI experiment: The imaging results in a number of improvements over conventional radiography, the inner tissue including the kidney vessel and the glomeruli were demonstrated.

Blood Vessel On IOXI study, the vasculars from the centre to the distal ( 5 grades) without administration of any contrast agent were clearly seen on the whole mouse kidney view image(see fig 2). The vessels with $30 \mu \mathrm{m}$ as much in diameter, could be identified. However, it is virtually impossible to observe blood vessels by conventional X-ray imaging techniques even if any contrast agent is injected. It is well known that the conventional X-ray systems, including CT, are incapable of visualizing vessels with a diameter less than $200 \mu \mathrm{m}$.

Imaging of renal microstructures In the imaging process, many new fine anatomic structures of the mouse kidney, such as the renal glomerulus-the structural and functional unit, could be clearly identified on the images (fig 3-5), including the shape, size and distribultion(in the coetex and medulla). Moreover, the space resolution could reach $10 \mu \mathrm{m}$ by measurement. It has been well known that the conventional image obtained by absorption contrast could not demonstrate such small inner structures at all. 


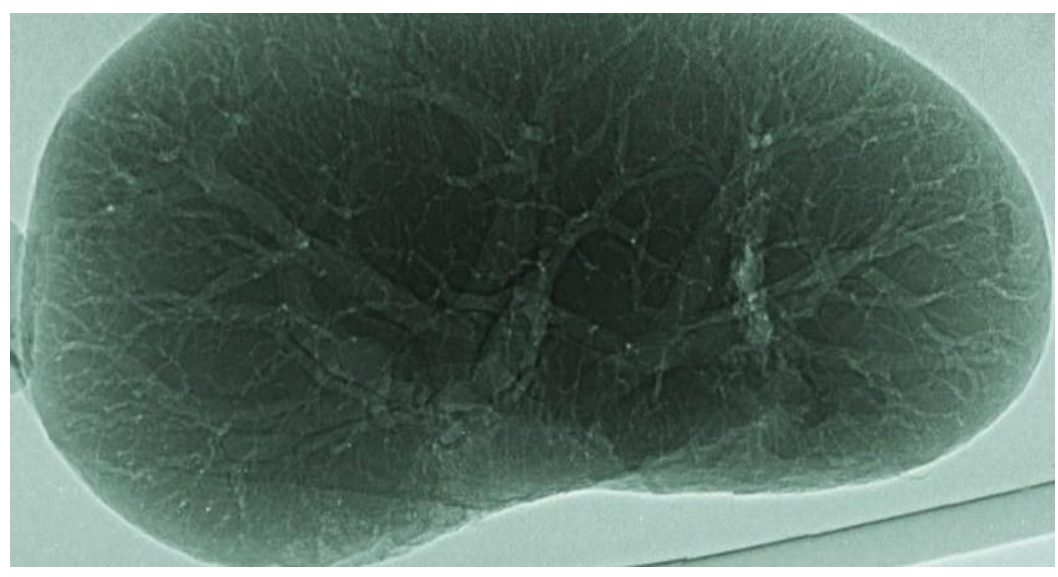

Fig3: Whole mouse kidney IOXI. The vessels from the hilum to the peripheral region are clearly revealed without using any contrast media.

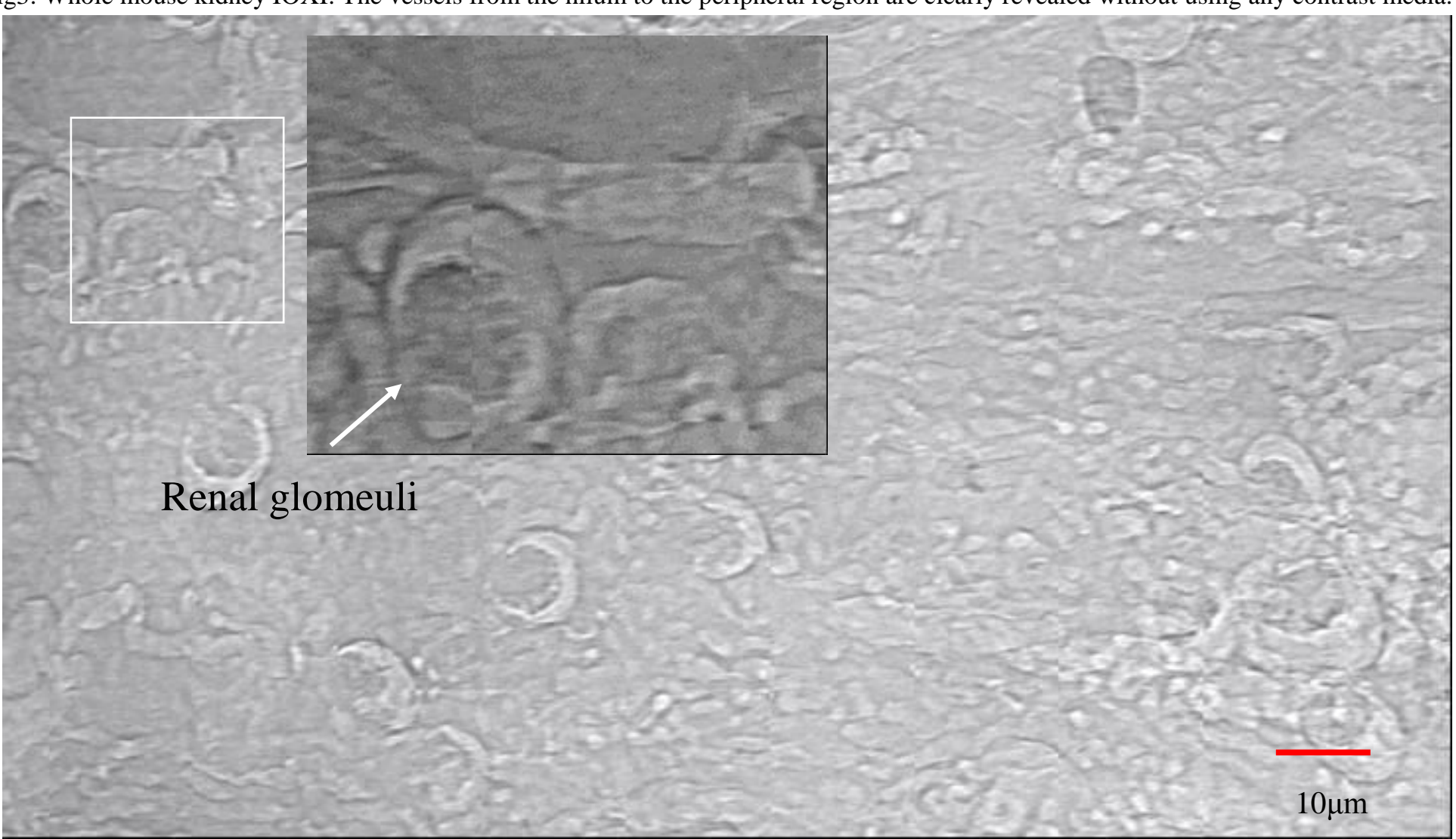

Fig4: magnified local slice imaging $(\times 20)$ and local magnified mouse kidney imaging $(\times 40)$. The glomeruli, tubules, and collecting ducts demonstated,

2) DEI experiment: In the imaging process, firstly the rocking curve which represents the relationship between the reflected intensity and deflection angle of the analyser was obtained by tuning the analyser (Fig 5). Then the DEI is performed at difference position on the rocking curve, typically including peak, waist and bottom position. Absorption imaging and DEI including low and high position in rocking curve images are obtained respectively. In the mouse kidney DEI imaging, the contrast in the waist imaging of high angle side (E) is highest(Fig6). The renal capsule is clear and compact, and the interface of cortex and medulla composed of arcuate vessels can be distinguished very well, The branches of the inter-lobular vessels almost reaching the edge of cortex with about $40 \mu \mathrm{m}$ diameter can be distinguished. However, due to lack of other information we can't separate from artery from venous, and no more other structure revealed on the imagings. 


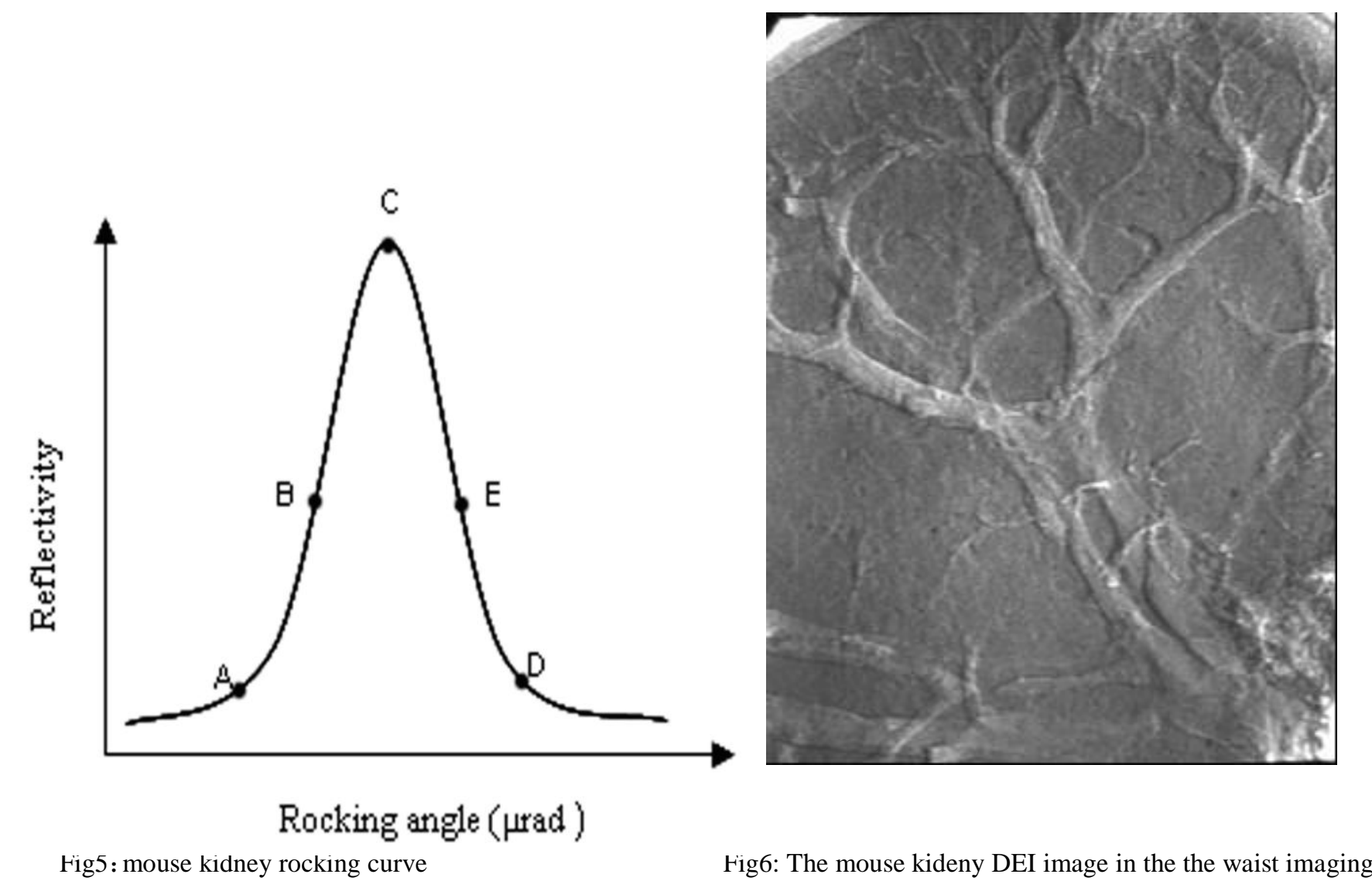

high angle side (E)

\section{Discussion}

The phase-contrast $\mathrm{x}$-ray imaging is of great value, the coherent X-ray source just like the Synchrotron radiation allows contrast to be improved by exploiting phase changes of the electromagnetic waves as they traverse tissues with different refractive indices, which provides $\mathrm{X}$-rays with intense, vertically collimated, polarized and continuous over a wide energy range. These highly desirable qualities allow a wide variety of imaging research to be performed at a synchrotron radiation.

Just like any other light source, the SR X-rays which has the nature of wave, and the wave shifts when X-ray pass through an object which is called $\mathrm{x}$-ray phase shift. The IOXI is a kind of x-ray imaging techniques for detecting the x-ray phase shift. In fact, in addition to absorption, several interactions, such as scatter, refraction, extinction, etc may occur as the X-ray photons traverse the subject being exposed. Besides the coherent light source, another influence elements on the IOXI is the distance $Z$ between the sample and detector ${ }^{[13]}$. The scattered beam in the forward direction (z-direction) interferes with the unscattered beam, thereby changing the amplitude of the propagating beam. When there are variations in the projected electron density $\rho(\mathrm{x}, \mathrm{y})$ of the object, variations of intensity are observed at the image plane. The contrast is proportional to the second derivative of $\rho(\mathrm{x}, \mathrm{y})$,so that interfaces and boundaries are enhanced in the images. The beam must be transversely coherent over the detail being imaged, but longitudinal coherence is not important so that even polychromatic radiation can be used ${ }^{[14]}$.

It is very important for the kidney imaging study, not only the vessels (including microvasculature) imaging in practice, but also more microstrutcture information. However, due to imaging quality limitation or harm diagnosis technique (like biopsy), no perfect technique has be used to do by now. The phase contrast imaging especially the IOXI has great potential to solve. It is promising that the renal glomerulus revealed clearly without any adminstration of contrast media in the IOXI experiment. The renal glomerulus is the basical structure and functional unit in the kidney which indicated disease and processing, however at present there is no imaging which makes the renal glomerulus visible, the clearly shown renal glomerulus indicated it is relatively easy for animal experiment and even clinical practice to find normal and abnormal, judge disease healing and progress, decide pre-treatment and following- up, and it is essential for diagnosis and treatment in time, delaying and ignoring may result in disaster.

The DEI is another imaging technique, When an analysing crystal with a narrow angular acceptance is placed in the transmitted beam new contrast mechanisms become important. The density of the object changes causes a change in the propagation direction of the incident beam. The diffracted images are essentially scatter free, since the crystal prevents much of the scatter from reaching the detector. The deviation of the beam is strongest when the density gradient is perpendicular to the beam. Therefore refraction contrast imaging is well suited for morphological studies of soft tissues. The refraction image is shown to have high sensitivity for delineating the boundaries of those regions in the object which have different refractive indices, and it is hoped that refraction by their contours can provide the contrast that is needed for imaging them. Besides refraction at the edges of the larger structures, strong small-angle scattering may be raised. These effects combine to enhance the visible contrast. However, due to the different nature of the contrast process, kideny imaging obtained with DEI appear very different and complex. It is not only straight forward to simulate or interpret DEI images, but also to identify lesions and differentiate them from the normal tissue in the future. In the DEI, we found that the microstructure was mainly vessel structure, and no other any more structure was noted, maybe due to the contrast is strongest at the edges of the vessels, while at the edge of other structures that are too small to produce visible contrast. However another view point 
out priority of the DEI is that it is adapt to thick tissue ${ }^{[15]}$. Constant improvement should be done about the kidney DEI imaging, not only theory but also imaging parameter. recently. Cheol Yong Yoon[12] use unmonochromatized hard X-ray in kidney imaging in the refractive index radiology system of PLS, clearly showed histological information of organelles in normal kidney such as glomeruli, tubules, and collecting ducts.

Many patent application of the PCI is being explored. For example in tumour study, imaging of vessels is very important to understand the vascular distribution within the organs and tumours. Currently the angiography systems by using conventional X-ray imaging with an X-ray tube cannot provide images of arteries less than $200 \mu \mathrm{m}$ in diameter. The possibility of making angiography by using phase contrast is evaluated with a physiological material composed of light elements. Recently, new research about phase contrast contrast agent has been lauched which will make the phase contrast angiography more useful towards more advanced imaging ${ }^{[16]}$.

Is the phase contrast imaging safe when it is used in vivo practice? It has been well known that the absorption contrast falls off quickly at higher energies E-4, being proportional to E-4[17], whereas the phase contrast is proportional to E-2 ${ }^{[17]}$. Thereby, imaging can be done at higher energies without significant loss of contrast, while largely reducing the radiation dose delivered to the tissue ${ }^{[18]}$ and the radiation dose will reduce greatly after monochromatization. Hence, phase-contrast imaging is a promising technique for observing and differentiate biological tissues.

In conclusion, It seems to be an ideal method for kidney imaging with the PCI, the IOXI in theses can reveal more minute structures like small vessels, however the DEI in the BSRF was mainly good at vessels (the comparison only limit to the two facility). The knowledge of these information will surely further improve our understanding of the physiological and pathological processes of biology tissue in animals. Although it is still a long way away from the imaging requirement for clinical imaging, further improvement in the SR phase contrast imaging including the facility and technique will enable more diseases visible, such as tumour and artery sclerosis, in their early stages.

Acknowledgements

This work is supported by the Projection of Shanghai Science Foundation, PRC (Grant No.11ZR1427800).

\section{Reference:}

[1]Arfelli Assante M, Bonvicini et a1. Low-dose phase contrast x-ray medical imaging. Phys Med Biol, 1998, 43: 2845 -2852.

[2] James R W 1962 The Optical Principles of the Diffraction of X-rays (London: Bell \& Sons) ch 4

[3]A.Momose,T. Takeda, Y. Itai,,K. Hirano, phase-contrast X-ray computed tomography for observing biological soft tissues, Nat.Med.2(1996)473.

[4]Momose A, Fukuda J. Phase-contrast radiographs of nonstained rat cerebellar specimen. Med Phys. 1995;22:375-379.

[5]. Takeda T, Momose A, Itai Y et al. Phase-contrast imaging with synchrotron X-rays for detecting cancer lesions. Acad Radiol 1995; 2:799-803.

[6]Fitzgerald R. Phase-sensitive X-ray imaging; new approaches that candetect X-ray phase shifts within soft tissues show promise for clinical and biological applications. Phys Today 2000; 53: 23-28.

[7]D Chapmanyz, W Thomlinsonx, R E Johnstonk, et al. Diffraction enhanced x-ray imaging[J] Phys Med. Biol. 42 (1997) $2015-2025$.

[8]Spanne P, Raven C, Snigireva I, et a1. In—line holography and phase contrast microtomography with high energy $\mathrm{x}$ - rays[J]. Phys Med Biol, 1999, 44(3): 741-749.

[9]A. Momose, Demonstration of phase contrast X-ray computed tomography using an $\mathrm{x}$-ray interferometer[J]. Nuclear instruments and Methods in Physics Research A1995 352:622-628.

[10] Jin $\mathrm{Wu}$, Tohoru Takeda, Thet Thet Lwin, et al Imaging renal structures by X-ray phase-contrast microtomography[J] Kidney International, (2009)75,945-951.

[11] Li Gang, Chen Zhihua, Wu Ziyu,et al. Image quality dependence on thickness of sliced rat kidney taken by a simplest DEI construction [J] Nuclear Instruments and Methods in Physics Research A 548 (2005) 200-206

[12] Cheol Yong Yoon, Duck Je Sung, Ju Han Lee, et al. Imaging of renal and prostate carcinoma with refractive index radiology International Journal of Urology(2007)14,96-103.

[13] Zhou, S.-A. \& Brahme, A. Development of phase-contrast X-ray imaging techniques and potential medical applications [J]. Physica Medica, 2008, 24: 129-148.

[14]PoganyA, GaoD and WilkinsSW, et al.Contrast and resolutionin imaging with a microfocus $\mathrm{x}$-ray source Rev.Sci.Instrum(1997).682:774-82.

[15] Li J, Zhong Z, Lidtke R, et al. Radiography of soft tissue of the foot and ankle with diffraction enhanced imaging. Journal of anatomy [J]. 2003, 202(5):463-470

[16] Christopher M Laperle, Theron J Hamilton, Philip Wintermeyer,et al. Low density contrast agents for x-ray phase contrast imaging: the use of ambient air for X-ray angiography of excised murine liver tissue[J]. Physics in medicine and biology. 2008, 53: 6911-6923.

[17]Dilmanian FA. Zhong Z. Ren B, etal Computed tomography of x-ray index of refraction using the diffraction enhanced imaging method. Physics in Medicine \& Biology. 2000, 45(4):933-46.

[18]J.keyrilāinen,M. Fernández,S. Fiedler,A. Bravin,ML. Karjalainen-Lindsberg,P. Virkkunen,E.M.Elo, M.Tenhuen,P.Suortti,W.Thomlinson, Visualisation of calcifications and thin collagen strands in human breast tumour specimens by the diffraction-enhanced imaging technique: a comparison with conventional mammography and histology, Eur.J. Radiol.53(2005)226. 